\title{
Effect of Area-Level Socioeconomic Deprivation on Risk of Cognitive Dysfunction in Older Adults
}

\author{
Adrian McCann, PhD, *Helene McNulty, PhD, *iD Jan Rigby, PhD, Catherine F. Hughes, PhD, * \\ Leane Hoey, PhD, * Anne M. Molloy, PhD, ${ }^{*}$ Conal J. Cunningham, MD, ${ }^{\S}$ Miriam C. Casey, MD, ${ }^{\S}$ \\ Fergal Tracey, MD," Maurice J. O’Kane, MD, ** Kevin McCarroll, MD, ${ }^{\S}$ Mary Ward, PhD,* \\ Katie Moore, BSc, * J.J. Strain, PhD, * and Adrian Moore, PhD ${ }^{+/}$
}

OBJECTIVES: To investigate the relationship between area-level deprivation and risk of cognitive dysfunction.

DESIGN: Cross-sectional analysis.

SETTING: The Trinity, Ulster, and Department of Agriculture (TUDA) study from 2008 to 2012.

PARTICIPANTS: Community-dwelling adults aged $74.0 \pm 8.3$ without dementia ( $\mathrm{N}=5,186 ; 67 \%$ female).

MEASUREMENTS: Adopting a cross-jurisdictional approach, geo-referenced address-based information was used to map and link participants to official socioeconomic indicators of deprivation within the United Kingdom and the Republic of Ireland. Participants were assigned an individual deprivation score related to the smallest administrative area in which they lived. These scores were categorized into comparable quintiles, that were then used to integrate the datasets from both countries. Cognitive health was assessed using the Mini-Mental State Examination (MMSE); cognitive dysfunction was defined as a MMSE score of 24 or less.

RESULTS: Approximately one-quarter of the cohort resided within the most-deprived districts in both countries. Greater area-level deprivation was associated with significantly lower MMSE scores; fewer years of formal education; greater anxiety, depression, smoking and

From the *Nutrition Innovation Centre for Food and Health, Ulster University, Coleraine, Northern Ireland, United Kingdom; ${ }^{\dagger}$ National Centre for GeoComputation, Maynooth University, Maynooth, Ireland;

Institute of Molecular Medicine, School of Medicine, Trinity College Dublin, Dublin, Ireland; ${ }^{\S}$ Mercer's Institute for Research on Ageing, St James's Hospital, Dublin, Ireland; "Causeway Hospital, Northern Health and Social Care Trust, Coleraine, Northern Ireland, United Kingdom;

* Clinical Chemistry Laboratory, Altnagelvin Hospital, Western Health and Social Care Trust, Londonderry, Northern Ireland, United Kingdom; and ${ }^{\dagger \dagger}$ School of Environmental Sciences, Ulster University, Coleraine, Northern Ireland, United Kingdom.

Address correspondence to Professor Helene McNulty, Nutrition Innovation Centre for Food and Health, Ulster University, Coleraine, Northern Ireland BT52 1SA, United Kingdom.

E-mail: h.mcnulty@ulster.ac.uk

See related editorial by Soo Borson.

DOI: $10.1111 /$ jgs. 15258 alcohol use, and obesity; and more adverse outcomes, including higher blood pressure and diabetes risk. After adjustment for relevant covariates, area deprivation was associated with significantly higher risk of cognitive dysfunction (odds ratio $=1.40,95 \%$ confidence interval $=1.05-1.87, P=.02$, for most vs least deprived).

CONCLUSION: This analysis combining data from two health systems shows that area deprivation is an independent risk factor for cognitive dysfunction in older adults. Adults living in areas of greatest socioeconomic deprivation may benefit from targeted strategies aimed at improving modifiable risk factors for dementia. Further cross-national analysis investigating the impact of arealevel deprivation is needed to address socioeconomic disparities and shape future policy to improve health outcomes in older adults. J Am Geriatr Soc 2018.

Key words: older adults; cross-jurisdictional; georeferencing; area-level deprivation; cognition

$\mathrm{H}$ ealth, well-being, and socioeconomic status are closely linked, with considerable evidence showing poorer health ${ }^{1}$ and earlier morbidity and death ${ }^{2-4}$ in persons at lower socioeconomic levels. There has been growing interest in whether the area in which a person lives can influence health in addition to the effects of individual socioeconomic factors. ${ }^{5}$ Area deprivation indices represent a geographic area-based composite measure of the socioeconomic deprivation of neighborhoods. They are typically constructed from a range of domains relating to deprivation such as income, employment, education, proximity to services, living environment, and crime and disorder and are presented as a single value or score for each neighborhood or area. Higher index values represent greater levels of deprivation in an area, and available evidence suggests that higher area deprivation is associated with greater risk 
of chronic disease and premature mortality ${ }^{6,7}$ independent of individual socioeconomic circumstances. ${ }^{6,8-10}$

Cognitive function in aging has become a global public health priority because it has important implications for independence and quality of life of older adults. ${ }^{11}$ With the prevalence of dementia predicted to triple by 2050 , it is important to identify individuals at greatest risk of developing cognitive dysfunction, an early predictor of dementia. ${ }^{11} \mathrm{Few}$ previous studies have examined the influence of area-level deprivation on cognitive health in older adults, although one study from England reported that greater area-level deprivation was not significantly associated with cognitive impairment and dementia after accounting for individual-level factors. ${ }^{12}$ To our knowledge, no previous study has investigated area-level deprivation in relation to cognitive health in cross-national research.

Some progress has been made in advancing the development of standardized deprivation indicators at a European Union level ${ }^{13}$ and more detailed comparisons of small area-level deprivation for a selection of countries within Europe, ${ }^{14}$ demonstrating the potential for using such measures in cross-national health inequality research. The aim of this study was to investigate the effect of arealevel socioeconomic deprivation on the risk of cognitive dysfunction and related health and lifestyle factors in older adults by combining data from two jurisdictions within the island of Ireland: Northern Ireland (NI), United Kingdom, and the Republic of Ireland (RoI).

\section{METHODS}

\section{Participants and Study Design}

This investigation was conducted as an observational study using data from the Trinity, Ulster, and Department of Agriculture (TUDA) cohort, as described in detail elsewhere. ${ }^{15}$ The TUDA study included 5,186 communitydwelling, noninstitutionalized adults aged 60 and older recruited between 2008 and 2012 from NI and RoI and initially sought to investigate the role of nutritional and related lifestyle factors in the development of three common diseases of aging - cardiovascular disease, osteoporosis, and dementia. TUDA participants were recruited using standardized protocols from general practice or hospital outpatient clinics and deemed suitable if they (or their parents) were born on the island of Ireland and did not have a diagnosis of dementia. Participants who were able to provide informed consent were considered eligible. The final study cohort was composed of 2,093 participants recruited in NI and 3,093 in RoI.

The Office for Research Ethics Committees Northern Ireland granted ethical approval (reference 08/NIR03/113), with corresponding approvals from the Northern and Western Health and Social Care Trusts in NI and the Research Ethics Committee of St James Hospital and The Adelaide and Meath Hospital in Dublin.

\section{Health and Lifestyle Data}

A health and lifestyle questionnaire was administered to participants to collect general information relevant to medical history, medication use, smoking status, and alcohol consumption. Weight and height were recorded to the nearest $0.01 \mathrm{~kg}$ and $0.01 \mathrm{~m}$, respectively, using portable scales and stadiometer (Seca; Brosch Direct Ltd, Peterborough, United Kingdom), and body mass index (BMI; kg/ $\mathrm{m}^{2}$ ) was calculated. Waist and hip measurements were recorded to the nearest $0.1 \mathrm{~cm}$ using a flexible tape measure and standardized protocols. The Timed Up-and-Go (TUG) test was administered as a measure of functional mobility. ${ }^{16}$ Participants were asked to stand from a seated position (seat height approximately $46 \mathrm{~cm}$ ), walk $3 \mathrm{~m}$ at their usual pace, turn around, walk back to the chair, and sit down. No physical assistance was given, and the time taken from command "Go" to completion of the task was measured using a stopwatch. Blood pressure (BP) measurements were taken in accordance with standard operating procedures. In brief, two measurements were taken from the reference arm (the arm with the highest BP reading), with a 5- to 10-minute interval between each measurement, and the mean of the two values was used as the BP value. Nonfasting blood samples were collected, stored, and analyzed using standard operating procedures and routine hospital laboratory assays.

Cognitive health was assessed using the Mini-Mental State Examination (MMSE), ${ }^{17}$ a short, structured cognitive test that evaluates global cognitive function by assessing the domains of orientation, registration, attention and concentration, recall and language and is the most widely used screening tool in clinical settings worldwide for identifying cognitive impairment or dementia. The maximum score achievable is 30 , with a score less than 25 indicating a possibility of cognitive impairment and a score less than 20 indicating dementia. Anxiety and depression were also assessed, using the Hospital Anxiety and Depression Scale ${ }^{18}$ and the Center for Epidemiologic Studies Depression Scale, respectively. ${ }^{19}$

\section{Measurement of Area Deprivation}

Deprivation indices are used in the United Kingdom and Ireland on a factor analytical approach that reduces a large number of indicator variables to a smaller number of underlying domains or factors that are presented as a single value or score. Participants from NI were initially mapped using their house number, street name, unit postcode, town information, and the Land and Property Services Ordnance Survey of Northern Ireland POINTER Geo-referencing database. ${ }^{20}$ After cleaning and verification of the address information, 1,982 participants (94.7\%) were geo-referenced and linked to an area deprivation score based on the Census Output Area (COA) in which they lived using data from the Northern Ireland Multiple Deprivation Measure 2010, ${ }^{21}$ which comprises 7 domains of deprivation, each developed to measure a distinct form or type of deprivation: income, employment, health, education, proximity to services, living environment, and crime. These domains were then presented as a single value or area deprivation score, which was then categorized according to quintile (each quintile representing $20 \%$ of all COAs in NI) ranging from least (Q1) to most (Q5) deprived. This was the preferred measure of deprivation because it was calculated at the smallest area-level 
available (with a mean number of households of 125 and a mean population of 340).

In the absence of a comparable postal code reference system in the RoI, an alternative geocoding method using Irish Grid $\mathrm{X}$ and $\mathrm{Y}$ co-ordinates ${ }^{22}$ was used to map and link participants to the appropriate socioeconomic indicators of area-level deprivation using the 2011 Pobal HP Deprivation Index for Small Areas in the Republic of Ireland, ${ }^{23}$ which conceptualizes underlying indicators of deprivation based on earlier deprivation indices for Ireland and analyses from other countries to identify three domains of affluence or disadvantage: demographic profile, social class composition, and labor market situation. After address information was cleaned and verified, 3,066 participants $(99.1 \%)$ were allocated to a Small Area Population Statistics (SAPS) area (with a mean number of households of 107 and mean population of 248) and given an area deprivation score. Again, the single area deprivation scores were categorized according to quintile, with each quintile representing $20 \%$ of all SAPS areas in RoI, ranging from least (Q1) to most (Q5) deprived.

Using local area deprivation data and the appropriate geo-referencing methods for each jurisdiction, comparable area deprivation scores categorized as quintiles could be generated, allowing for TUDA study data from two different countries within the island of Ireland (NI, RoI) to be effectively linked and integrated. One hundred thirty-eight $(2.7 \%)$ participants of the study cohort were not allocated an area deprivation quintile because of incomplete address information.

\section{Statistical Analysis}

Statistical analysis was performed using SPSS version 23.0 (IBM Corp., Armonk, NY). Before statistical analysis was performed, tests for normality were performed, and variables were log-transformed as appropriate. Between-group differences were analyzed using analysis of covariance (controlling for relevant covariates) with Bonferroni correction for multiple comparisons or chi-square tests using standardized residuals. The effect of area deprivation on the main study outcome, cognitive dysfunction, was investigated using logistic regression. In line with current clinical practice in the United Kingdom and Ireland, participants were classified according to MMSE score into two groups - normal cognitive function (MMSE score $\geq 25$, reference) and cognitive dysfunction (MMSE score $\leq 24$ ). Area deprivation was categorized into quintiles, and the model was adjusted for factors relevant to cognitive dysfunction. These covariates included age, sex, education, depression, anxiety, BMI, TUG test, smoking, alcohol, blood pressure, blood lipids, and diabetes.

\section{RESULTS}

Relevant characteristics of the TUDA study cohort are shown in Table 1. Of the 5,186 TUDA study participants, $5,048(97.3 \%)$ were allocated to an area deprivation quintile. Figure 1 shows the geographical distribution of TUDA participants in NI and RoI according to area deprivation category. Marginally higher proportions of TUDA participants were found in the least- $(21.2 \%)$ and most- $(26.2 \%)$ deprived quintiles than in the other quintiles.

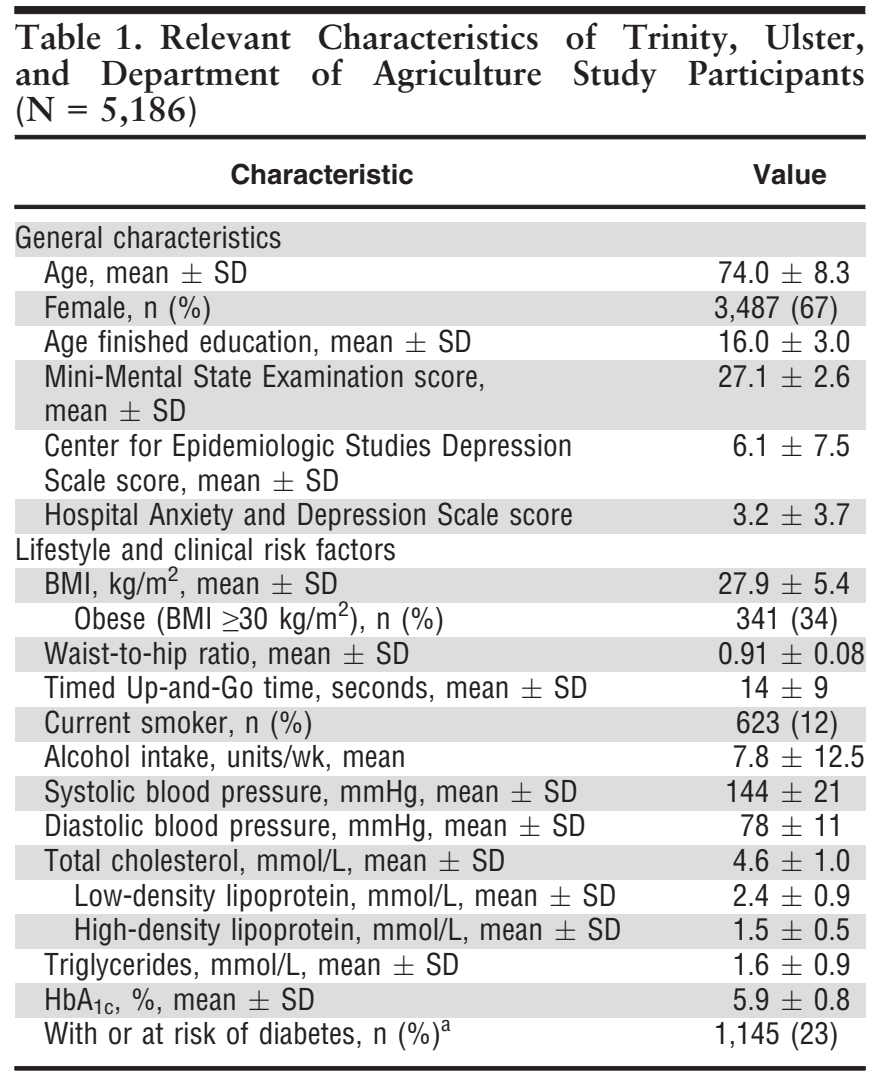

$\mathrm{SD}=$ standard deviation; $\mathrm{BMI}=$ body mass index.

${ }^{\mathrm{a}}$ Glycosylated hemoglobin $\left(\mathrm{HbA}_{1 \mathrm{c}}\right) \geq 6.1 \%$ or antidiabetic medication use.

Participant characteristics and disease risk factors were then examined in relation to area-level socioeconomic deprivation (Table 2). MMSE score was significantly lower at the highest levels of area deprivation (Q4, Q5). With increasing area deprivation, the number of years spent in formal education decreased, and anxiety, depression, smoking, alcohol use, and obesity all increased, along with disease risk factors, including blood pressure and diabetes risk.

Table 3 shows determinants of cognitive dysfunction in older Irish adults, calculated using logistic regression analysis. Area-level socioeconomic deprivation was associated with greater risk of cognitive dysfunction (odds ratio $=1.40 ; \quad 95 \% \quad$ confidence $\quad$ interval $=1.05-1.87$; $P=.02$ for most vs least deprived) after adjustment for other relevant factors. A comparison of logistic regression analysis with and without area deprivation showed that the inclusion of area deprivation significantly strengthened the model (likelihood ratio test; see Table 3 footnote).

\section{DISCUSSION}

This study showed that area-level socioeconomic deprivation in older people is associated with poor cognition and an adverse general health profile. Older adults living in areas with the greatest socioeconomic deprivation in NI and the RoI had a $40 \%$ greater risk of cognitive dysfunction than those living in areas of least deprivation, after adjustment for other relevant risk factors.

There is accumulating evidence that the place where a person lives influences their disease risk, even after 


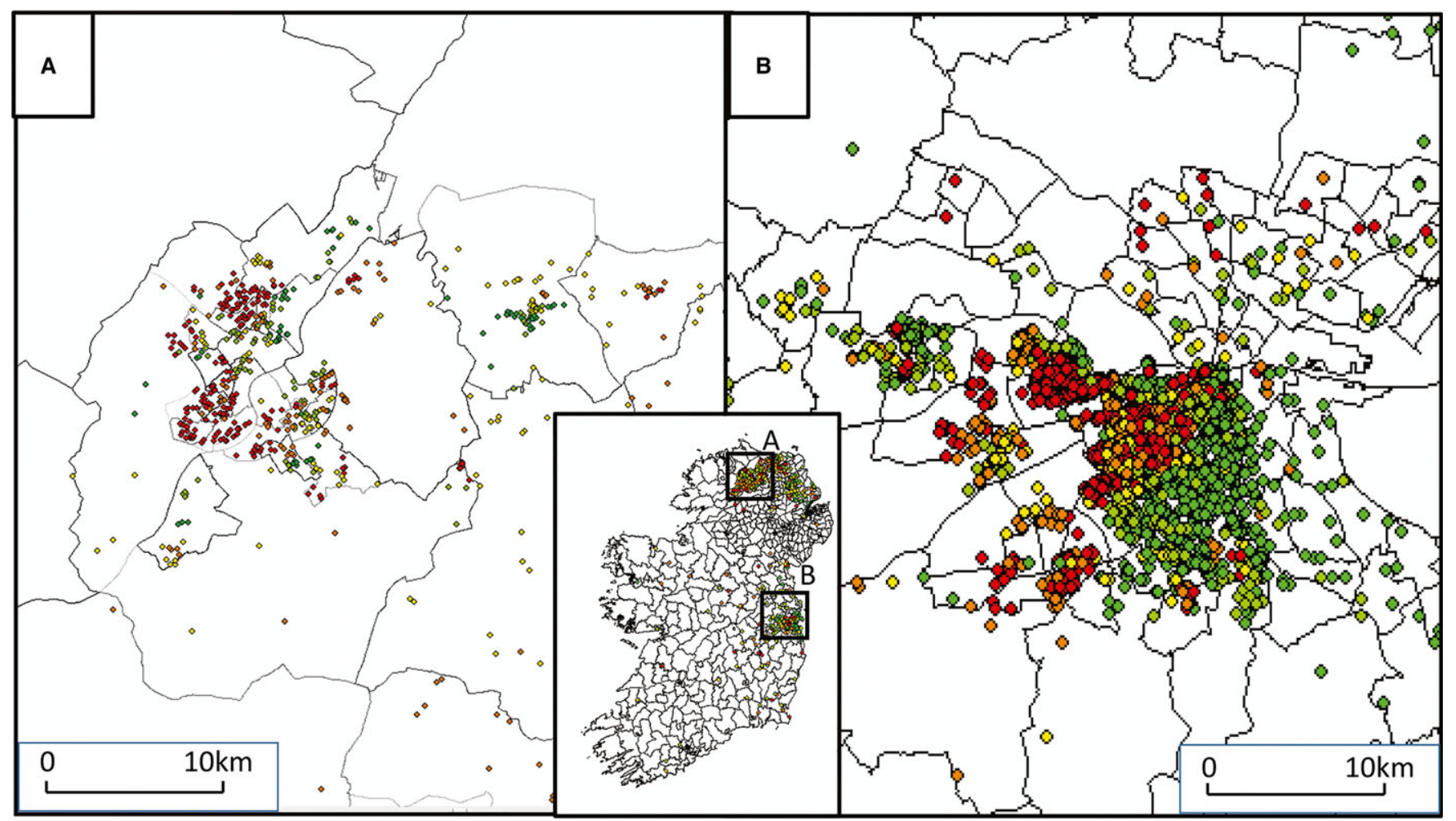

Figure 1. Map showing the distribution of Trinity, Ulster, and Department of Agriculture study participants from (A) Northern Ireland, United Kingdom and (B) the Republic of Ireland, color-coded according to area deprivation category, ranging from least-deprived $20 \%$ of areas (GREEN) to most-deprived $20 \%$ of areas (RED) in each jurisdiction.

accounting for individual factors. ${ }^{6-10,24-26}$ Area deprivation refers to a geographical measure of the socioeconomic deprivation in an area or region. In the current cross-jurisdictional study, greater area deprivation was associated with significantly lower MMSE scores, fewer years spent in formal education, poorer mental health, higher alcohol use, and smoking (e.g., $8 \%$ vs $18 \%$ smokers in least vs most deprived), along with a greater range of disease risk factors, notably blood pressure, diabetes risk, and obesity (e.g., $27 \%$ vs $35 \%$ obese in least vs most deprived). These observations are generally consistent with findings from other studies undertaken in Sweden, Australia, England, and the United States. ${ }^{27-30}$ Most previous studies investigating area-based deprivation have focused on mortality ${ }^{7}$ and cardiovascular diseases or diabetes, ${ }^{6,31}$ and there is a paucity of research investigating the influence of area deprivation on cognitive dysfunction; in particular, no previous study has addressed this relationship across different jurisdictions. In the current study, consistent with previous reports, ${ }^{32,33}$ older people living in areas of higher deprivation were found to have higher rates of depression and anxiety. Poor mental health (in particular depression) is in turn an established risk factor for cognitive dysfunction, and the current results showed that depression was an independent factor contributing to cognitive dysfunction.

The mechanisms underlying the relationship between area deprivation and cognitive dysfunction in the present study are not clear, but educational attainment ${ }^{34}$ and depression $^{35}$ may be two important mediators in this complex relationship. One recent population-based longitudinal survey of more than 10,000 older adults in the
United States concluded that higher educational attainment was associated with significantly lower risk of dementia. $^{36}$ Likewise, a 2-decade comparison of the prevalence of dementia in the United Kingdom suggests that education is a strong modifiable factor in dementia, ${ }^{37}$ further supporting the view that poor education may be one of the drivers of the relationship between area deprivation and cognition shown here. We found significantly lower educational attainment in older adults living in areas of greatest deprivation, who had 3 fewer years of formal education than those in the least deprived areas. In addition, depression has been shown to increase cognitive dysfunction, and poorer cognitive health can also predispose older adults to depression, ${ }^{38,39}$ suggesting a bidirectional relationship between the two conditions. Also, in agreement with the current findings, it was previously observed that depression in older adults was associated with socioeconomic disadvantage, poverty, and deprivation related to place of residence. ${ }^{40}$ The association between area deprivation and cognitive dysfunction observed in the current study remained significant even after adjustment for education, depression, and other factors, suggesting that other social determinants of health could also contribute to this relationship. These include contextual poverty, income inequality, social cohesion, access to resources, and relationships with the built and natural environment. $^{1,12,41}$

Despite expectations that aging populations globally would lead to large increases in the number of adults with dementia, recent studies from the United States and Europe suggest that the prevalence of dementia in some 
Table 2. Participant Characteristics and Disease Risk Factors According to Quintile (Q) of Area Deprivation

\begin{tabular}{|c|c|c|c|c|c|c|}
\hline Characteristic & Q1, $n=1,069$ & Q2, $\mathrm{n}=859$ & Q3, $n=919$ & Q4, $\mathrm{n}=\mathbf{8 7 7}$ & $Q 5, n=1,324$ & $P$-Value \\
\hline \multicolumn{7}{|l|}{ General characteristics } \\
\hline Age, mean $\pm S D$ & $75.4 \pm 8.7$ & $74.0 \pm 8.3$ & $73.3 \pm 7.9$ & $73.6 \pm 8.0$ & $73.9 \pm 8.3$ & $<.001$ \\
\hline Female, $n(\%)$ & $734(69)$ & $585(68)$ & $591(64)$ & $597(68)$ & $886(67)$ & .36 \\
\hline Age finished education, mean $\pm S D$ & $17.8 \pm 3.8$ & $16.6 \pm 3.1$ & $15.9 \pm 2.7$ & $15.3 \pm 2.2$ & $14.8 \pm 1.9$ & $<.001$ \\
\hline Mini-Mental State Examination score, mean \pm SD & $27.4 \pm 2.5$ & $27.4 \pm 2.3$ & $27.4 \pm 2.3$ & $26.9 \pm 2.7$ & $26.5 \pm 2.8$ & .008 \\
\hline $\begin{array}{l}\text { Center for Epidemiologic Studies Depression } \\
\text { Scale score, mean } \pm \text { SD }\end{array}$ & $5.2 \pm 6.8$ & $5.4 \pm 6.8$ & $5.5 \pm 7.4$ & $6.3 \pm 7.6$ & $7.5 \pm 8.2$ & .001 \\
\hline Hospital Anxiety and Depression Scale score & $2.7 \pm 3.2$ & $2.8 \pm 3.4$ & $3.1 \pm 3.6$ & $3.4 \pm 3.8$ & $3.7 \pm 4.0$ & .01 \\
\hline \multicolumn{7}{|l|}{ Lifestyle and clinical risk factors } \\
\hline $\mathrm{BMI}, \mathrm{kg} / \mathrm{m}^{2}$, mean $\pm \mathrm{SD}$ & $27.1 \pm 5.0$ & $27.5 \pm 5.1$ & $28.0 \pm 5.1$ & $28.7 \pm 5.8$ & $28.1 \pm 5.8$ & $<.001$ \\
\hline Obese $\left(\mathrm{BMI} \geq 30 \mathrm{~kg} / \mathrm{m}^{2}\right), \mathrm{n}(\%)$ & $291(27)$ & $274(32)$ & $324(35)$ & $348(40)$ & $469(35)$ & $<.001$ \\
\hline Waist-to-hip ratio, mean $\pm \mathrm{SD}$ & $0.90 \pm 0.08$ & $0.90 \pm 0.09$ & $0.91 \pm 0.08$ & $0.92 \pm 0.09$ & $0.91 \pm 0.08$ & .001 \\
\hline Timed Up-and-Go time, seconds, mean \pm SD & $14 \pm 10$ & $14 \pm 9$ & $13 \pm 9$ & $14 \pm 9$ & $15 \pm 9$ & .009 \\
\hline Current smoker, $\mathrm{n}(\%)$ & $86(8)$ & $76(9)$ & $100(11)$ & $108(12)$ & $242(18)$ & $<.001$ \\
\hline Alcohol intake, units/wk, mean & $6.6 \pm 10.9$ & $6.7 \pm 10.9$ & $8.0 \pm 12.8$ & $8.1 \pm 12.9$ & $9.3 \pm 14.5$ & .004 \\
\hline Systolic blood pressure, $\mathrm{mmHg}$, mean $\pm \mathrm{SD}$ & $142 \pm 20$ & $144 \pm 21$ & $147 \pm 21$ & $145 \pm 21$ & $144 \pm 21$ & .006 \\
\hline Diastolic blood pressure, $\mathrm{mmHg}$, mean $\pm \mathrm{SD}$ & $77 \pm 11$ & $78 \pm 11$ & $79 \pm 11$ & $79 \pm 11$ & $78 \pm 12$ & .03 \\
\hline Total cholesterol, $\mathrm{mmol} / \mathrm{L}$, mean $\pm \mathrm{SD}$ & $4.7 \pm 1.0$ & $4.7 \pm 1.0$ & $4.7 \pm 1.1$ & $4.6 \pm 1.1$ & $4.6 \pm 1.0$ & .01 \\
\hline Low-density lipoprotein, mmol/L, mean $\pm \mathrm{SD}$ & $2.5 \pm 0.9$ & $2.4 \pm 0.8$ & $2.5 \pm 0.9$ & $2.4 \pm 0.9$ & $2.4 \pm 0.9$ & .06 \\
\hline High-density lipoprotein, $\mathrm{mmol} / \mathrm{L}$, mean $\pm \mathrm{SD}$ & $1.6 \pm 0.5$ & $1.5 \pm 0.5$ & $1.4 \pm 0.5$ & $1.4 \pm 0.5$ & $1.4 \pm 0.5$ & $<.001$ \\
\hline Triglycerides, $\mathrm{mmol} / \mathrm{L}$, mean $\pm \mathrm{SD}$ & $1.5 \pm 0.8$ & $1.5 \pm 0.9$ & $1.7 \pm 0.9$ & $1.6 \pm 0.9$ & $1.6 \pm 0.9$ & .001 \\
\hline $\mathrm{HbA}_{1 \mathrm{c}}, \%$, mean $\pm \mathrm{SD}$ & $5.8 \pm 0.8$ & $5.8 \pm 0.8$ & $5.8 \pm 0.8$ & $6.0 \pm 0.9$ & $5.9 \pm 0.8$ & .20 \\
\hline With or at-risk of diabetes, $\mathrm{n}(\%)^{\mathrm{a}}$ & $209(20)$ & 157 (18) & $189(21)$ & $244(28)$ & 315 (24) & .001 \\
\hline
\end{tabular}

Participants were allocated to a deprivation quintile (1-5) based on the deprivation score of the area in which they lived, with 1 being the least deprived and 5 the most deprived.

Statistical tests: Between-group analysis of covariance (controlling for age) with Bonferroni correction for multiple comparisons or chi-square test using standardized residuals. $P<.05$ was considered significant.

$\mathrm{SD}=$ standard deviation; $\mathrm{BMI}=$ body mass index .

${ }^{\mathrm{a}}$ Glycosylated hemoglobin $\left(\mathrm{HbA}_{1 \mathrm{c}}\right) \geq 6.1 \%$ or antidiabetic medication use.

Table 3. Determinants of Cognitive Dysfunction in Older Adults $(\mathbf{n}=4,554)$

\begin{tabular}{|c|c|c|c|}
\hline Determinant & Beta Value & OR (95\% Confidence Interval) & $P$-Value \\
\hline Age & 0.059 & $1.06(1.05-1.08)$ & $<.001$ \\
\hline Female sex & -0.078 & $0.93(0.74-1.15)$ & .49 \\
\hline Center for Epidemiologic Studies Depression Scale score & 0.040 & $1.04(1.03-1.06)$ & $<.001$ \\
\hline Hospital Anxiety and Depression Scale score & -0.016 & $0.98(0.96-1.01)$ & .27 \\
\hline Body mass index & -0.024 & $0.98(0.96-1.00)$ & .02 \\
\hline Alcohol consumption in past year & 0.084 & $1.09(0.83-1.43)$ & .54 \\
\hline Systolic blood pressure & -0.002 & $1.00(0.99-1.00)$ & .52 \\
\hline Diastolic presssure & 0.003 & $1.00(0.99-1.01)$ & .50 \\
\hline Total cholesterol & -0.025 & $0.98(0.88-1.08)$ & .64 \\
\hline Triglycerides & -0.074 & $0.93(0.81-1.06)$ & .28 \\
\hline With or at risk of diabetes ${ }^{a}$ & 0.023 & $1.02(0.81-1.29)$ & .85 \\
\hline 4 & 0.209 & $1.23(0.89-1.70)$ & .20 \\
\hline 5 & 0.337 & $1.40(1.05-1.87)$ & .02 \\
\hline
\end{tabular}

Cognitive dysfunction defined as Mini-Mental State Examination (MMSE) score $\leq 24$.

Results obtained from a fully adjusted logistic regression model.

Odds ratio (OR) given as exponentiation of the beta coefficient $(\operatorname{Exp} B)$, where $\operatorname{Exp} B$ = change in the OR).

Likelihood ratio test indicates a significant improvement in the logistic regression model with the inclusion of area deprivation (with area deprivation: loglikelihood $=2,907.8$, chi-square $=519.2$; degrees of freedom $(d f)=20, P<.001$; without area deprivation: $\log$-likelihood $=2,926.7$; chi-square $=500.4$, $d f=16, P<.001)$.

${ }^{\mathrm{a}}$ Glycosylated hemoglovin $\geq 6.1 \%$ or antidiabetic medication use.

${ }^{\mathrm{b}}$ Based on the score of the area in which participants live (reference Q1: least deprived). 
countries may be stabilizing (or even declining), possibly as a result of improved health in midlife and potential protection that better educational attainment in early life affords. ${ }^{36,37}$ The findings of a recent investigation of epidemiological data over time from 5 studies in western Europe suggest that primary prevention aimed at increasing cognitive reserve, along with better treatment of vascular and chronic conditions, could have the greatest effect on future dementia. ${ }^{37}$ In addition, deprived social environments are known to breed social isolation and psychosocial stress and limit access to resources and health services, all of which can potentially interact with individual susceptibility to cognitive dysfunction. The current findings linking area deprivation with not only greater risk of cognitive dysfunction and lower educational attainment, but also a range of adverse lifestyle and cardiovascular disease risk factors, points to the living environment as an important component in dementia risk and thus a worthwhile target for efforts to reduce dementia occurrence and disability. A comprehensive report recently highlighted the potential for effective dementia prevention through targeted interventions to modify risk factors that could transform the future for society. ${ }^{42}$

The current study benefited from the use of a large, well-characterized cohort of older adults. It used individual-level data on health and disease status and area-level data on deprivation to determine whether living in a deprived area increases the risk of poor health, specifically cognitive dysfunction. A further strength was the use of novel country-specific geocoding approaches that facilitated the integration of regionally independent ethnically homogenous (Caucasian) datasets, enabling the TUDA cohort as a whole to be readily described in relation to the underlying socioeconomic profile of the base population of the two countries within the island of Ireland. This novel cross-jurisdictional approach provided a unique opportunity to link area-level deprivation with cognitive outcomes in older adults from two health systems; to our knowledge, this is the first time this has been achieved. As such, this work sets a precedent for future research initiatives seeking to integrate comparable data from cross-national studies (e.g., Survey of Health, Ageing and Retirement in Europe $^{43}$ ) to investigate area-level deprivation in relation to health. The limitations of our approach relate to geographical coverage, geo-referencing accuracy, scale, and the direct comparability of measures of deprivation across jurisdictions. The study also used unique composite measures of deprivation from each jurisdiction made up of different combinations of univariate socioeconomic indicators; thus the measures of small area deprivation are not exactly comparable, but for the purposes of this study, the measures were categorized into quintiles ranging from the most- to the least-deprived areas in each jurisdiction, providing a meaningful measure for comparison across the deprivation spectrum. In addition, although area-based income deprivation indicators are associated with health outcomes, the effect is less pronounced than that of individual income measures, ${ }^{44}$ and as such, the lack of specific data relating to personal income has a potentially confounding residual influence in this analysis. Nevertheless, this study further underscores the value of using area deprivation indices (that include domains related to personal income and wealth), particularly in situations in which socioeconomic data for individuals are not readily available. Finally, although the MMSE as a measure of cognition has sometimes been criticized for its ceiling and floor effects, it is the most widely used screening cognitive test in clinical settings and in epidemiological studies worldwide. $^{45}$

In conclusion, the novel, cross-jurisdictional approach of the current study provides a unique insight into the relationship between area deprivation and cognitive performance and suggests that older Irish adults living in areas with the greatest level of deprivation are at significantly higher risk $(40 \%)$ of cognitive dysfunction. Although further research is needed to fully elucidate the mechanisms explaining our observations, this work represents a first step toward identifying the specific aspects of area-level socioeconomic deprivation connected with cognitive health in older adults. Given the widening health and socioeconomic disparities seen globally, the current findings identify the potential for effective dementia prevention through targeted interventions to modify risk factors in communities with the greatest area-level socioeconomic deprivation.

\section{ACKNOWLEDGMENTS}

The TUDA study was supported by government funding from the Irish Department of Agriculture, Food and the Marine and the Health Research Board (under its Food Institutional Research Measure) and from the Northern Ireland Department for Employment and Learning (under its strengthening the all-Ireland research base initiative). Funding to support the geo-referencing methodologies described in this article was obtained through a small grant from the Centre for Ageing Research and Development in Ireland and further facilitated by the Health Research Board of Ireland (HRA_PHS/2010/19).

Conflict of Interest: None declared.

Author Contributions: AM, HMN, LH, AMM, JR, AMC: study concept and design, funding. AMC, AM, JR: data analysis. HMN, AM, JR, AMC, CFH, LH, KM, AMM, CC, MCC, FT, MOK, KMC, MW, JJS: data interpretation. AMC, AM, CFH, HMN: drafting the manuscript. All authors: critical revision of manuscript for important intellectual content, approval of content. HMN and AM had full access to the data and take responsibility for the data analysis.

Sponsor's Role: The funders of this research had no role in the design or conduct of the study, data collection, management, analysis, or preparation of the paper.

\section{REFERENCES}

1. Lee $\mathrm{Y}$, Back JH, Kim J et al. Multiple socioeconomic risks and cognitive impairment in older adults. Dement Geriatr Cogn Disord 2010;29:523529.

2. Adler N, Singh-Manoux A, Schwartz J et al. Social status and health: A comparison of British civil servants in Whitehall-II with European- and African-Americans in CARDIA. Soc Sci Med 2008;66:1034-1045.

3. Braveman PA, Cubbin C, Egerter $S$ et al. Socioeconomic disparities in health in the United States: What the patterns tell us. Am J Public Health 2010;100(Suppl 1):S186-S196.

4. Zimmer Z, Hanson HA, Smith KR. Childhood socioeconomic status, adult socioeconomic status, and old-age health trajectories: Connecting early, middle, and late life. Demogr Res 2016;34:285-320. 
5. Pickett KE, Pearl M. Multilevel analyses of neighbourhood socioeconomic context and health outcomes: A critical review. J Epidemiol Community Health 2001;55:111-122.

6. Wild S, Macleod F, McKnight J, et al. Impact of deprivation on cardiovascular risk factors in people with diabetes: An observational study. Diabet Med 2008;25:194-199.

7. Connolly S, O'Reilly D, Rosato M. House value as an indicator of cumulative wealth is strongly related to morbidity and mortality risk in older people: A census-based cross-sectional and longitudinal study. Int J Epidemiol 2010;39:383-391.

8. Lawlor DA, Davey Smith G, Patel R et al. Life-course socioeconomic position, area deprivation, and coronary heart disease: Findings from the British Women's Heart and Health Study. Am J Public Health 2005;95:91-97.

9. Wight RG, Aneshensel CS, Miller-Martinez D, et al. Urban neighborhood context, educational attainment, and cognitive function among older adults. Am J Epidemiol 2006;163:1071-1078.

10. Lang IA, Llewellyn DJ, Langa KM et al. Neighborhood deprivation, individual socioeconomic status, and cognitive function in older people: Analyses from the English Longitudinal Study of Ageing. J Am Geriatr Soc 2008;56:191-198.

11. Prince M, Wimo A, Guerchet M et al. World Alzheimer's Report-The Global Impact of Dementia. London, UK: Alzheimer's Disease International, 2015.

12. Wu YT, Prina AM, Jones AP, et al. Community environment, cognitive impairment and dementia in later life: Results from the Cognitive Function and Ageing Study. Age Ageing 2015;44:1005-1011.

13. Guio AM, Marlier E, Gordon D et al. Improving the measurement of material deprivation at the European Union level. J Eur Soc Pol 2016;26:219-233.

14. Guillaume E, Pornet C, Dejardin O, et al. Development of a cross-cultural deprivation index in five European countries. J Epidemiol Community Health 2016;70:493-499.

15. McCarroll K, Beirne A, Casey M, et al. Determinants of 25-hydroxyvitamin D in older Irish adults. Age Ageing 2015;44:847-853.

16. Podsiadlo D, Richardson S. The timed "Up \& Go": A test of basic functional mobility for frail elderly persons. J Am Geriatr Soc 1991;39:142148.

17. Folstein MF, Folstein SE, McHugh PR. 'Mini-mental state'. A practical method for grading the cognitive state of patients for the clinician. J Psychiatr Res 1975;12:189-198.

18. Zigmond AS, Snaith RP. The Hospital Anxiety and Depression Scale. Acta Psychiatr Scand 1983;67:361-370.

19. Radloff LS. The CES-D Scale: A self report depression scale for research in the general population. Appl Psychol Meas 1977;1:385-401.

20. Land \& Property Services. Ordnance Survey of Northern Ireland (LPS OSNI) POINTER Geo-referencing database [on-line]. Available at https://data.gov.uk/dataset/lps-osni-pointer-download Accessed October 10, 2013.

21. Northern Ireland Multiple Deprivation Measure 2010 (NIMDM2010) [online]. Available at https://www.nisra.gov.uk/statistics/deprivation/northernireland-multiple-deprivation-measure-2010-nimdm2010 Accessed October 12, 2013.

22. Rigby JE, Boyle M, Brunsdon C, et al. Towards a geography of health inequalities in Ireland. Irish Geography 2017;50:37-58.

23. Pobal HP. 2011. Deprivation Index for Small Areas in the Republic of Ireland [on-line]. Available at https://maps.pobal.ie/WebApps/DeprivationInd ices/index.html Accessed January 20, 2014.

24. Marmot M. Social determinants of health inequalities. Lancet 2005;365:1099-1104.

25. Chetty R, Stepner M, Abraham S, et al. The association between income and life expectancy in the United States, 2001-2014. JAMA 2016;315:1750-1766.
26. Beuscart JB, Genin M, Dupont C, et al. Potentially inappropriate medication prescribing is associated with socioeconomic factors: A spatial analysis in the French Nord-Pas-de-Calais Region. Age Ageing 2017;46:607-613.

27. Cubbin C, Sundquist K, Ahlen $\mathrm{H}$ et al. Neighborhood deprivation and cardiovascular disease risk factors: Protective and harmful effects. Scand J Public Health 2006;34:228-237.

28. Adams RJ, Howard N, Tucker G, et al. Effects of area deprivation on health risks and outcomes: A multilevel, cross-sectional, Australian population study. Int J Public Health 2009;54:183-192.

29. Tabassum F, Breeze E, Kumari M. Coronary heart disease risk factors and regional deprivation in England: Does age matter? Age Ageing 2010;39:253-256.

30. Laraia BA, Karter AJ, Warton EM et al. Place matters: Neighborhood deprivation and cardiometabolic risk factors in the Diabetes Study of Northern California (DISTANCE). Soc Sci Med 2012;74:1082-1090.

31. Avendano M, Kunst AE, Huisman M, et al. Socioeconomic status and ischaemic heart disease mortality in 10 western European populations during the 1990s. Heart 2006;92:461-467.

32. Walters K, Breeze E, Wilkinson P et al. Local area deprivation and urbanrural differences in anxiety and depression among people older than 75 years in Britain. Am J Public Health 2004;94:1768-1774.

33. Aneshensel CS, Wight RG, Miller-Martinez D et al. Urban neighborhoods and depressive symptoms among older adults. J Gerontol B Psychol Sci Soc Sci 2007;62B:S52-S59.

34. Reuser M, Willekens FJ, Bonneux L. Higher education delays and shortens cognitive impairment: A multistate life table analysis of the US Health and Retirement Study. Eur J Epidemiol 2011;26:395-403.

35. van den Kommer TN, Comijs HC, Aartsen MJ et al. Depression and cognition: How do they interrelate in old age? Am J Geriatr Psychiatry 2013;21:398-410.

36. Langa KM, Larson EB, Crimmins EM, et al. A comparison of the prevalence of dementia in the united states in 2000 and 2012. JAMA Intern Med 2017;177:51-58.

37. Wu YT, Fratiglioni L, Matthews FE, et al. Dementia in Western Europe: Epidemiological evidence and implications for policy making. Lancet Neurol 2016;15:116-124.

38. Panza F, Frisardi V, Capurso C, et al. Late-life depression, mild cognitive impairment, and dementia: Possible continuum? Am J Geriatr Psychiatry 2010;18:98-116.

39. Brailean A, Aartsen MJ, Muniz-Terrera G, et al. Longitudinal associations between late-life depression dimensions and cognitive functioning: A crossdomain latent growth curve analysis. Psychol Med 2017;47:690-702.

40. Kim D. Blues from the neighborhood? Neighborhood characteristics and depression Epidemiol Rev 2008;30:101-117.

41. Fisher M, Baum F. The social determinants of mental health: Implications for research and health promotion. Aust N Z J Psychiatry 2010;44:10571063.

42. Livingston G, Sommerlad A, Orgeta V, et al. Dementia prevention, intervention, and care. Lancet 2017;390:2673-2734.

43. Borsch-Supan A, Brandt M, Hunkler C, et al. Data resource profile: The Survey of Health, Ageing and Retirement in Europe (SHARE). Int J Epidemiol 2013;42:992-1001.

44. Siegel M, Mielck A, Maier W. Individual income, area deprivation, and health: Do income-related health inequalities vary by small area deprivation? Health Econ 2015;24:1523-1530.

45. Franco-Marina F, Garcia-Gonzalez JJ, Wagner-Echeagaray F, et al. The Mini-Mental State Examination revisited: Ceiling and floor effects after score adjustment for educational level in an aging Mexican population. Int Psychogeriatr 2010;22:72-81. 